\title{
STABILIZATION FOR THE TRANSMISSION PROBLEM OF THE TIMOSHENKO SYSTEM IN THERMOELASTICITY WITH TWO CONCENTRATED MASSES
}

\author{
WAEL YOUSSEF
}

\begin{abstract}
In this paper, our main goal is to study the stability of the thermoelastic Timoshenko beam with locally distributed temperature. Then, we consider the transmission problem of the Timoshenko system in thermoelasticity with two concentrated masses. We show the non-exponential stability by using a result introduced by J. E. Muñoz Rivera and R. Racke [18] based on the Weyl theorem. Otherwise, we prove the polynomial stability by using a frequency domain method.
\end{abstract}

\section{INTRODUCTION}

The suppression of vibration of elastic structures is one of the important topics in the material science. One interesting problem is the stability of solutions of wave, elastic and thermoelastic equations. To stabilize the oscillations in the solutions of wave equations, different types of dissipation mechanisms have been introduced to work either on the domain, part of it, or at a portion of the boundary. In this paper, we are interested for a model which defines the oscillation of a plate which is composed of a purely elastic part connected by a vibrating concentrated masses with two another thermoelastic parts (see Fig. 1). Thereby we have material with localized thermoelastic effect.

In this work, we shall study the transmission problem for the following Timoshenko beam which is composed of three components, one elastic and two thermoelastic. It is governed by the following partial differential equations:

$$
\left\{\begin{array}{l}
\rho_{1} \varphi_{t t}-k\left(\varphi_{x}+\psi\right)_{x}=0 \quad \text { in }(0, L) \backslash \Theta \times(0, \infty), \\
\rho_{2} \psi_{t t}-b \psi_{x x}+k\left(\varphi_{x}+\psi\right)=0 \quad \text { in }(0, L) \backslash \Theta \times(0, \infty), \\
\delta_{1} \Phi_{t t}-k_{1}\left(\Phi_{x}+\Psi\right)_{x}=0 \quad \text { in } \Theta \times(0, \infty), \\
\delta_{2} \Psi_{t t}-b_{1} \Psi_{x x}+k_{1}\left(\Phi_{x}+\psi\right)+\alpha \theta_{x}=0 \quad \text { in } \Theta \times(0, \infty), \\
\theta_{t}-\theta_{x x}+\alpha \Psi_{x t}=0 \quad \text { in } \Theta \times(0, \infty) \\
\left(\Phi, \Phi_{t}, \Psi, \Psi_{t}, \theta\right)(x, 0)=\left(\Phi^{0}, \Phi^{1}, \Psi^{0}, \Psi^{1}, \theta^{0}\right)(x), \\
\left(\varphi, \varphi_{t}, \psi, \psi_{t}\right)(x, 0)=\left(\varphi^{0}, \varphi^{1}, \psi^{0}, \psi^{1}\right)(x),
\end{array}\right.
$$

Date: September 3, 2019

Key words and phrases. Thermoelasticity, Timoshenko beam; Transmission problem. 
with $t>0,0<x<L$, and $\Theta:=] 0, c[\cup] d, L\left[\right.$, where $0<c<d<L . \rho_{1}, \rho_{2}, k, b, \delta_{1}$, $\delta_{2}, k_{1}$, and $b_{1}$ are positive physical constants. $\alpha$ is the coupling parameter.

$\begin{array}{lll}\text { Termoelastic part } & \text { elastic part } & \text { Termoelastic part }\end{array}$

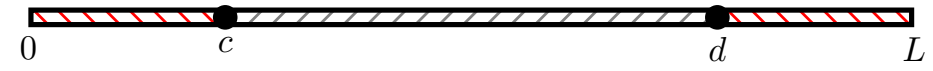

The system satisfies the following boundary conditions

$$
\Phi(t, x)=\Psi(t, x)=\theta(t, x)=0, x=0, L
$$

and the following transmission conditions

$$
\left\{\begin{array}{l}
k \varphi(t, x)=k_{1} \Phi(t, x), b \psi(t, x)=b_{1} \Psi(t, x) \quad(x=c, d) \\
\theta_{x}(x)=0 \quad(x=c, d) \\
-M \Phi_{t t}(c, t)=k_{1}\left(\Phi_{x}+\Psi\right)(c, t)-k\left(\varphi_{x}+\psi\right)(c, t) \\
-M \Psi_{t t}(c, t)=b_{1} \Psi_{x}(c, t)-\alpha \theta(c, t)-b \psi_{x}(c, t) \\
N \Phi_{t t}(d, t)=k_{1}\left(\Phi_{x}+\Psi\right)(d, t)-k\left(\varphi_{x}+\psi\right)(d, t) \\
N \Psi_{t t}(d, t)=b_{1} \Psi_{x}(d, t)-\alpha \theta(d, t)-b \psi_{x}(d, t)
\end{array}\right.
$$

where $M, N>0$. The energy of solutions of the system (1.1)-(1.3) is defined by

$$
\mathcal{E}(t)=\mathcal{E}_{1}(t)+\mathcal{E}_{2}(t)+\frac{M}{2}\left|\Phi_{t}(c, t)\right|^{2}+\frac{M}{2}\left|\Psi_{t}(c, t)\right|^{2}+\frac{N}{2}\left|\Phi_{t}(d, t)\right|^{2}+\frac{N}{2}\left|\Psi_{t}(c, t)\right|^{2},
$$

where

$$
\mathcal{E}_{1}(t)=\frac{1}{2} \int_{c}^{d}\left(\rho_{1}\left|\varphi_{t}\right|^{2}+\rho_{2}\left|\psi_{t}\right|^{2}+b\left|\psi_{x}\right|^{2}+k\left|\varphi_{x}+\psi\right|^{2}\right) d x
$$

and

$$
\mathcal{E}_{2}(t)=\frac{1}{2} \int_{\Theta}\left(\delta_{1}\left|\Phi_{t}\right|^{2}+\delta_{2}\left|\Psi_{t}\right|^{2}+b_{1}\left|\Psi_{x}\right|^{2}+k_{1}\left|\Phi_{x}+\Psi\right|^{2}+|\theta|^{2}\right) d x .
$$

Now, let us multiply the first equation of (1.1) by $\varphi_{t}$ and the second by $\psi_{t}$, then integration over $(c, d)$. Again, we multiply the equation by $\Phi_{t}$ and the fourth by $\Psi_{t}$, then integration over $\Theta$. Thus, by using the boundary conditions (1.2) and the transmission conditions (1.3) we get

$$
\frac{d}{d t} \mathcal{E}(t)=-\int_{\Theta}\left|\theta_{x}\right|^{2} d x \leq 0
$$

which called the dissipation relation.

Recently, the stabilization of the transmission problems have attracted vast interest. In [7] Z-J Han and G-Q. Xu investigated a transmission problem between elastic and thermoelastic materials which are connected by a vibrating concentrated mass. They proved that this system is not exponentially stable, but they establish a polynomial stability with estimation of the optimal decay. C.A. Raposo et al. [20] studied a transmission problem for the Timoshenko beam by considering the case when a part of the beam has friction and the other is purely elastic. They proved the exponential decay for the solution. In [14], S. A. Messaoudi and B. Said-Houari established the exponential stability of the solution of a $1 D$ linear thermoelastic 
transmission problem, where the heat conduction is described by the theories of Green and Naghdi. J. E. Munõz Rivera and R. Racke [18] considered transmission problems between a (thermo)viscoelastic system with KelvinVoigt damping, and a purely elastic system. They proved that neither the elastic damping by KelvinVoigt mechanisms nor the dissipative effect of the temperature in one material can assure the exponential stability of the total system when it is coupled through transmission to a purely elastic system. By using Weyls theorem on perturbations of the essential spectrum. They showed the lack of exponential stability. Moreover, they proved the polynomial stability by using an extension of a result Borichev and Tomilov [3]. Also, we refer the readers for some other results on the transmission problems $[1,13,19,22]$ and on the thermoelasticity $[2,10,15,16,21]$.

This paper is organized as follows. In section 2 we prove the well posedness of the system (1.1)-(1.3) by formulating an appropriate Hilbert state space setting. In Section 3 the nonexponential stability is established. Section 4 is devoted to the polynomial stability.

\section{WeLl-Posedness}

First, we need to introduce the following spaces:

$$
\mathcal{H}_{1}:=\left\{\begin{array}{c}
(\varphi, \psi, \Phi, \Psi, \theta) \in\left(H^{1}(] c, d[)\right)^{2} \times\left(H^{1}(\Theta)\right)^{3} \\
\Phi(t, x)=\Psi(t, x)=\theta(t, x)=0, x=0, L \\
k \varphi(t, x)=k_{1} \Phi(t, x), b \psi(t, x)=b_{1} \Psi(t, x), \quad(x=c, d) \\
\theta_{x}(x)=0, \quad(x=c, d)
\end{array}\right\}
$$

and

$$
\mathcal{H}:=\mathcal{H}_{1} \times \mathcal{L}^{2} \times \mathbb{C}^{4}
$$

Now, consider the bilinear form $h$ defined over $\mathcal{H} \times \mathcal{H}$ by:

$$
\left\{\begin{aligned}
h\left(W_{1}, W_{2}\right) & :=\int_{c}^{d}\left[\rho_{1} u_{1} \overline{u_{2}}+\rho_{2} v_{1} \overline{v_{2}}+b \psi_{1 x} \overline{\psi_{2 x}}+k\left(\varphi_{1 x}+\psi_{1} \overline{\left(\varphi_{2 x}+\psi_{2}\right.}\right)\right] d x \\
& +\int_{\Theta}\left[\delta_{1} p_{1} \overline{p_{2}}+\delta_{2} q_{1} \overline{q_{2}}+b_{1} \Psi_{1 x} \overline{\Psi_{2 x}}+k_{1}\left(\Phi_{1 x}+\Psi_{1} \overline{\left(\Phi_{2 x}+\Psi_{2}\right)}+\theta_{1} \overline{\theta_{2}}\right] d x\right. \\
& +M z_{1} \overline{z_{2}}+M w_{1} \overline{w_{2}}+N r_{1} \overline{r_{2}}+N y_{1} \overline{y_{2}} .
\end{aligned}\right.
$$

for all

$$
W_{1}:=\left(\varphi_{1}, \psi_{1}, \Phi_{1}, \Psi_{1}, \theta_{1}, u_{1}, v_{1}, p_{1}, q_{1}, z_{1}, w_{1}, r_{1}, y_{1}\right) \in \mathcal{H}
$$

and

$$
W_{2}:=\left(\varphi_{2}, \psi_{2}, \Phi_{2}, \Psi_{2}, \theta_{2}, u_{2}, v_{2}, p_{2}, q_{2}, z_{2}, w_{2}, r_{2}, y_{2}\right) \in \mathcal{H}
$$


Then, for $W:=(\varphi, \psi, \Phi, \Psi, \theta, u, v, p, q, z, w, r, y) \in \mathcal{H}$, we have

$$
\begin{aligned}
h(W, W)=0 \Rightarrow & \int_{c}^{d}\left[\rho_{1}|u|^{2}+\rho_{2}|v|^{2}+b\left|\psi_{x}\right|^{2}+k\left|\varphi_{x}+\psi\right|^{2}\right] d x \\
& +\int_{\Theta}\left[\delta_{1}|p|^{2}+\delta_{2}|q|^{2}+b\left|\Psi_{x}\right|^{2}+k\left|\Phi_{x}+\Psi\right|^{2}+|\theta|^{2}\right] d x \\
& +M|z|^{2}+M|w|^{2}+N|r|^{2}+N|y|^{2}=0 \\
\Rightarrow & u=v=0 \text { on }(c, d), \quad p=q=0 \quad \text { on } \Theta \\
& \Phi_{x}+\Psi=\Psi_{x}=\theta=0 \text { on } \Theta \\
& \psi_{x}=\varphi_{x}+\psi=0 \text { on }(c, d) .
\end{aligned}
$$

So $\Psi=$ cte on $\Theta$, but $\Psi(0)=0$. Hence $\Psi=0$ and so $\Phi_{x}=0$ on $\Theta$. Therefore, $\Phi=$ cte on $\Theta$. The fact that $\Phi(0)=0$ leads to $\Psi=0$ on $\Theta$.

On the other hand, by the transmission conditions, we have $b \psi(x)=b_{1} \Psi(x),(x=$ $c, d)$. Then $\psi=0$ on $(c, d)$ and we get that $\varphi=0$ on $(c, d)$ because $k \varphi(x)=$ $k_{1} \Phi(x),(x=c, d)$. Therefore, $W=0_{\mathcal{H}}$.

Consequently, $h$ is an positive-definite inner product of $\mathcal{H}$ associated to the energy norm. Thereby $\mathcal{H}$ is a Hilbert space under this norm.

Define the linear operator $\mathcal{A}$ by:

$$
D(\mathcal{A}):=\left\{\begin{array}{l}
(\varphi, \psi, \omega, \Phi, \Psi, \theta, u, v, p, q, z, w, r, y) \in \mathcal{H} / \\
(\varphi, \psi, \Phi, \Psi, \theta) \in \mathcal{H}_{1} \cap\left[\left(H^{2}(] c, d[)\right)^{2} \times\left(H^{2}(\Theta)\right)^{3}\right], \\
\left(u, v, p, q, \theta_{x x}-\alpha q_{x}\right) \in \mathcal{H}_{1}, z=p(c), w=q(c), r=p(d), y=q(d)
\end{array}\right\},
$$

and

$$
\mathcal{A} W=\left(\begin{array}{c}
u \\
v \\
p \\
q \\
\theta_{x x}-\alpha q_{x} \\
\frac{k}{\rho_{1}}\left(\varphi_{x x}+\psi_{x}\right) \\
\frac{b}{\rho_{2}} \psi_{x x}-\frac{k}{\rho_{2}}\left(\varphi_{x}+\psi\right) \\
\frac{k}{\delta_{1}}\left(\Phi_{x x}+\Psi_{x}\right) \\
\frac{b}{\delta_{2}} \Psi_{x x}-\frac{k}{\delta_{2}}\left(\Phi_{x}+\Psi\right)-\frac{\alpha}{\delta_{2}} \theta_{x} \\
-\frac{1}{M}\left[k_{1}\left(\Phi_{x}+\Psi\right)(c)-k\left(\varphi_{x}+\psi\right)(c)\right] \\
-\frac{1}{M}\left[b_{1} \Psi_{x}(c)-\alpha \theta(c)-b \psi_{x}(c)\right] \\
\frac{1}{N}\left[k_{1}\left(\Phi_{x}+\Psi\right)(d)-k\left(\varphi_{x}+\psi\right)(d)\right] \\
\frac{1}{N}\left[b_{1} \Psi_{x}(d)-\alpha \theta(d)-b \psi_{x}(d)\right]
\end{array}\right)
$$

Then the system (1.1) can be reformulated into an evolution problem of first order on $\mathcal{H}$ in the form

$$
\left\{\begin{array}{l}
W^{\prime}(t)=\mathcal{A} W(t), \quad t>0 \\
W(0)=W^{0} \in D(\mathcal{A})
\end{array}\right.
$$


Now, we are ready to summarize the well-posedness result in the following theorem.

Theorem 2.1. The operator $\mathcal{A}$ generates a $C^{0}$ - semigroup $S(t)$ of contractions on $\mathcal{H}$. In addition, for any initial data $W^{0} \in D(\mathcal{A}), W(t)$ is a strong solution of (2.1) i.e. $W(t) \in \mathcal{C}^{1}([0, \infty), \mathcal{H}) \cap \mathcal{C}^{0}([0, \infty), D(\mathcal{A}))$.

Proof. For $W=\left(\varphi, \psi, \Phi, \Psi, \theta, u, v, p, q, z_{1}, z_{2}, r_{1}, r_{2}\right) \in D(\mathcal{A})$, a straightforward calculations leads to

$$
\Re\langle\mathcal{A} W, W\rangle:=\Re h(\mathcal{A} W, W)=-\int_{\Theta}\left(\left|\theta_{x}\right|^{2}\right) d x \leq 0 .
$$

Next, let us prove that

$$
\mathcal{A} W=\left(f_{1}, f_{2}, \ldots, f_{9}, f_{10}, f_{11}, f_{12}, f_{13}\right)
$$

has a unique solution, for any $\left(f_{1}, f_{2}, \ldots, f_{13}\right) \in \mathcal{H}$.

In order, the first forth equations of (2.3) imply that

$$
u=f_{1}, v=f_{2}, p=f_{3}, q=f_{4} .
$$

From an other side, substituting of $q$ in the forth equation of (2.3) and using the standard elliptic PDE theory yield to the existence of the unique solution

$$
\theta \in\left(H_{c}^{1}(0, c) \cap H_{c}^{2}(0, c)\right)
$$

of the equation

$$
\left\{\begin{array}{l}
\theta_{x x}=\alpha f_{4 x}+f_{5} \\
\theta(0)=0, \quad \theta_{x}(c)=0 .
\end{array}\right.
$$

where, for $j \in \mathbb{N}^{*}$,

$$
H_{c}^{j}(0, c):=\left\{f \in H^{j}(0, c) / f(0)=0\right\} .
$$

Similarly, for the existence of the unique solution

$$
\theta \in\left(H_{d}^{1}(d, L) \cap H_{d}^{2}(d, L)\right),
$$

where

$$
H_{d}^{j}(d, L):=\left\{f \in H^{j}(d, L) / f(L)=0\right\} .
$$

It remains to establish the existence of $(\varphi, \psi, \Phi, \Psi)$ satisfying

$$
\begin{aligned}
k\left(\varphi_{x x}+\psi_{x}\right) & =\rho_{1} f_{6}, \\
b \psi_{x x}-\frac{k}{\rho_{2}}\left(\varphi_{x}+\psi\right) & =\rho_{2} f_{7}, \\
k_{1}\left(\Phi_{x x}+\Psi_{x}\right) & =\delta_{1} f_{8}, \\
b_{1} \Psi_{x x}-k_{1}\left(\Phi_{x}+\Psi\right) & =\delta_{2} \alpha \theta_{x}+\delta_{2} f_{9}, \\
k_{1}\left(\Phi_{x}+\Psi\right)(c)-k\left(\varphi_{x}+\psi\right)(c) & =-M f_{10}, \\
b_{1} \Psi_{x}(c)-b \psi_{x}(c) & =-M f_{11}-\alpha \theta(c), \\
k_{1}\left(\Phi_{x}+\Psi\right)(d)-k\left(\varphi_{x}+\psi\right)(d) & =N f_{12}, \\
b_{1} \Psi_{x}(d)-b \psi_{x}(d) & =N f_{13}+\alpha \theta(d) .
\end{aligned}
$$


For this claim, consider the continuous and coercive linear form

$$
\left\{\begin{array}{l}
\mathbb{B}((\varphi, \psi, \Phi, \Psi),(\widetilde{\varphi}, \widetilde{\psi}, \widetilde{\Phi}, \widetilde{\Psi})):=\int_{c}^{d}\left[b \psi_{x} \widetilde{\psi}_{x}+k\left(\varphi_{x}+\psi\right)\left(\widetilde{\varphi}_{x}+\widetilde{\psi}\right)\right] d x \\
\quad+\int_{\Theta}\left[b_{1} \Psi_{x} \widetilde{\Psi}_{x}+k_{1}\left(\Phi_{x}+\Psi\right)\left(\widetilde{\Phi}_{x}+\widetilde{\Psi}\right)\right] d x
\end{array}\right.
$$

for $(\varphi, \psi, \Phi, \Psi),(\widetilde{\varphi}, \widetilde{\psi}, \widetilde{\Phi}, \widetilde{\Psi})$ belong to the Hilbert space

$\mathcal{G}:=\left\{\begin{array}{c}(\varphi, \psi, \Phi, \Psi) \in\left(H^{1}(] c, d[)\right)^{2} \times\left(H^{1}(\Theta)\right)^{2} / \Phi(t, x)=\Psi(t, x)=0, x=0, L, \\ k \varphi(t, x)=k_{1} \Phi(t, x), b \psi(t, x)=b_{1} \Psi(t, x), \quad(x=c, d)\end{array}\right\}$.

Let $\mathbb{E}$ be the continuous linear form defined by

$$
\left\{\begin{array}{l}
\mathbb{F}(\widetilde{\varphi}, \widetilde{\psi}, \widetilde{\Phi}, \widetilde{\Psi}):=-\rho_{1} \int_{c}^{d} f_{6} \widetilde{\varphi} d x-\rho_{2} \int_{c}^{d} f_{7} \widetilde{\psi} d x-\delta_{1} \int_{\Theta} f_{8} \widetilde{\Phi} d x-\delta_{2} \int_{\Theta} f_{7} \widetilde{\Psi} d x \\
+N f_{12} \widetilde{\varphi}(d)+M f_{10} \widetilde{\varphi}(c)+\left(N f_{13}+\alpha \theta(d)\right) \widetilde{\psi}(d)+\left(N f_{11}-\alpha \theta(c)\right) \widetilde{\psi}(c) .
\end{array}\right.
$$

By applying the Lax-Milgram theorem, there exists a unique $(\varphi, \psi, \Phi, \Psi)$ in $\mathcal{G}$ such that

$$
\mathbb{B}((\varphi, \psi, \Phi, \Psi),(\widetilde{\varphi}, \widetilde{\psi}, \widetilde{\Phi}, \widetilde{\Psi}))=\mathbb{F}(\widetilde{\varphi}, \widetilde{\psi}, \widetilde{\Phi}, \widetilde{\Psi})
$$

for every $(\widetilde{\varphi}, \widetilde{\psi}, \widetilde{\Phi}, \widetilde{\Psi}) \in \mathcal{H}$. Therefore, the $(2.3)$ has a unique solution $W \in D(\mathcal{A})$ and so $0 \in \rho(\mathcal{A})$. Thus, by the resolvant identity, for $\lambda>0$, we have $R(\lambda I-\mathcal{A})=\mathcal{H}$ (see Theorem 1.2.4 in [12]). So, by applying the Lumer-Philips theorem the proof is achieved.

\section{Nonexponential stability}

We start this section by some notations and definitions. Let $X$ be a Banach space. A bounded operator $S \in \mathscr{L}(X)$ is called a Fredholm operator if there is $T \in \mathscr{L}(X)$ such that $\operatorname{Id}_{X}-T S$ and $\operatorname{Id}_{X}-S T$ are compact. We denote by

$$
\sigma_{\text {ess }}(S):=\mathbb{C} \backslash\left\{\lambda \in \mathbb{C} ; \lambda \operatorname{Id}_{X}-S \text { is a Fredholm operator }\right\}
$$

the essential spectrum of $S$. Moreover, we define the essential spectral radius by

$$
r_{\mathrm{ess}}(S):=\max \left\{|\lambda| ; \lambda \in \sigma_{\mathrm{ess}}(S)\right\} .
$$

For more details see [4], pp. 248-250.

The proof of the nonexponential stability is based on the following theorem proved by J. Rivera and R. Racke [18] which based on the Weyl theorem (theorem 3.3 page $3748)$.

Theorem 3.1. Let $\mathcal{H}_{1}$ be a Hilbert space with norm $\|\cdot\|_{\mathcal{H}_{1}}$, and let $\varnothing \nsubseteq \mathcal{H}_{2} \subset \mathcal{H}_{1}$ be a closed subspace with orthogonal projection $P: \mathcal{H}_{1} \longrightarrow \mathcal{H}_{2}$. Let $S_{j}=\left(S_{j}(t)\right)_{t \geq 0}$ be a $C_{0}$-semigroup on $\mathcal{H}_{j}$ for $j=1,2$. If there exists $t_{0}>0$ such that for $t \geq t_{0}>0$ we have

(1) $r_{\text {ess }}\left(S_{2}(t)\right) \geq 1$,

(2) $S_{1}(t)-S_{2}(t): \mathcal{H}_{2} \longrightarrow \mathcal{H}_{1}$ is compact,

then $S_{1}$ is not exponentially stable. 
Now, let us establish the following observability result which will plays an important role for the proof of the main theorem in this section.

Lemma 3.1. Let $T>0$. There exists a positive constant $C$ such that

$$
\left\{\begin{array}{l}
\int_{0}^{T}\left|\varphi_{t}(c)\right|^{2} d t+\int_{0}^{T}\left|\varphi_{t}(d)\right|^{2} d t \\
+\int_{0}^{T}\left|\left(\varphi_{x}+\psi\right)(c)\right|^{2} d t+\int_{0}^{T}\left|\left(\varphi_{x}+\psi\right)(d)\right|^{2} d t \leq C \mathcal{E}(0)
\end{array}\right.
$$

and

$$
\int_{0}^{T}\left|\psi_{t}(c)\right|^{2} d t+\int_{0}^{T}\left|\psi_{t}(d)\right|^{2} d t+\int_{0}^{T}\left|\left(\psi_{x}\right)(c)\right|^{2} d t+\int_{0}^{T}\left|\left(\psi_{x}\right)(d)\right|^{2} d t \leq C \mathcal{E}(0)
$$

Proof. Let $\Lambda \in \mathcal{C}^{1}([c, d])$ be a given function satisfying $\Lambda(c)=-\Lambda(d)=2 \gamma$, where $\gamma>0$. Multiplying the first equation of (1.1) by $\Lambda(x)\left(\varphi_{x}+\psi\right)$ and integrating the resulting equation over $[0, T] \times[c, d]$ we get

$$
\rho_{1} \int_{0}^{T} \int_{c}^{d} \varphi_{t t} \Lambda(x)\left(\varphi_{x}+\psi\right) d x-k \int_{0}^{T} \int_{c}^{d}\left(\varphi_{x}+\psi\right)_{x} \Lambda(x)\left(\varphi_{x}+\psi\right) d x=0
$$

Therefore,

$$
\left\{\begin{array}{l}
-\rho_{1} \int_{0}^{T} \int_{c}^{d} \Lambda(x) \varphi_{t}\left(\varphi_{x t}+\psi_{t}\right) d x d t+\rho_{1} \int_{c}^{d} \Lambda(x)\left[\varphi_{t}\left(\varphi_{x}+\psi\right)\right]_{0}^{T} d x \\
+k \int_{0}^{T} \int_{c}^{d} \frac{\Lambda_{x}}{2}\left|\varphi_{x}+\psi\right|^{2} d x d t-k \int_{0}^{T}\left[\frac{\Lambda(x)}{2}\left|\varphi_{x}+\psi\right|^{2}\right]_{c}^{d} d t=0
\end{array}\right.
$$

thus,

$$
\left\{\begin{array}{l}
\rho_{1} \int_{0}^{T} \int_{c}^{d} \frac{\Lambda_{x}}{2}\left|\varphi_{t}\right|^{2} d x d t-\rho_{1} \int_{0}^{T}\left[\frac{\Lambda(x)}{2}\left|\varphi_{t}\right|^{2}\right]_{c}^{d} d t \\
+\rho_{1} \int_{0}^{T} \int_{c}^{d} \varphi_{t} \psi_{t} d x d t+\rho_{1} \int_{c}^{d} \Lambda(x)\left[\varphi_{t}\left(\varphi_{x}+\psi\right)\right]_{0}^{T} d x \\
+k \int_{0}^{T} \int_{c}^{d} \frac{\Lambda_{x}}{2}\left|\varphi_{x}+\psi\right|^{2} d x d t-k \int_{0}^{T}\left[\frac{\Lambda(x)}{2}\left|\varphi_{x}+\psi\right|^{2}\right]_{c}^{d} d t=0
\end{array}\right.
$$

Hence,

$$
\left\{\begin{array}{l}
\rho_{1} \gamma \int_{0}^{T}\left|\varphi_{t}(c)\right|^{2} d t+\rho_{1} \gamma \int_{0}^{T}\left|\varphi_{t}(d)\right|^{2} d t \\
+k \gamma \int_{0}^{T}\left|\left(\varphi_{x}+\psi\right)(c)\right|^{2} d t+k \gamma \int_{0}^{T}\left|\left(\varphi_{x}+\psi\right)(d)\right|^{2} d t= \\
-\rho_{1} \int_{0}^{T} \int_{c}^{d} \varphi_{t} \psi_{t} d x d t-\rho_{1} \int_{c}^{d} \Lambda(x)\left[\varphi_{t}\left(\varphi_{x}+\psi\right)\right]_{0}^{T} d x \\
-k \int_{0}^{T} \int_{c}^{d} \frac{\Lambda_{x}}{2}\left|\varphi_{x}+\psi\right|^{2} d x d t
\end{array}\right.
$$

So, by the Cauchy-Shwarz inequality and the definition of $\mathcal{E}$ we get (3.12).

The estimation (3.13) is obtained by multiplying the second equation of (1.1) by $\Lambda(x)\left(\varphi_{x}+\psi\right)$ and repeating the same arguments to get (3.12). 
Now, define the semigroup $S_{0}$ by the following initial boundary value problem over $(0, L)$ :

$$
\left\{\begin{array}{l}
\rho_{1} \widetilde{\varphi}_{t t}-k\left(\widetilde{\varphi}_{x}+\widetilde{\psi}\right)_{x}=0 \quad \text { in }(0, L) \backslash \Theta \times(0, \infty), \\
\rho_{2} \widetilde{\psi}_{t t}-b \widetilde{\psi}_{x x}+k\left(\widetilde{\varphi}_{x}+\widetilde{\psi}\right)=0 \quad \text { in }(0, L) \backslash \Theta \times(0, \infty), \\
\left(\widetilde{\varphi}, \widetilde{\varphi}_{t}, \widetilde{\psi}, \widetilde{\psi}_{t}\right)(x, 0)=\left(\widetilde{\varphi}_{0}, \widetilde{\varphi}_{1}, \widetilde{\psi}, \widetilde{\psi}_{1}\right)(x) \quad \text { in }(0, L) \backslash \Theta
\end{array}\right.
$$

with boundary conditions

$$
\left\{\begin{array}{l}
\widetilde{\varphi}(t, x)=\widetilde{\psi}(t, x)=0 \quad(x=c, d) \\
\widetilde{\varphi}_{x}(x, t)=\widetilde{\psi}_{x}(x, t)=0 \quad(x=c, d) .
\end{array}\right.
$$

and initial condidtions

$$
\widetilde{\varphi}(0, x)=\widetilde{\varphi}_{0}, \widetilde{\psi}(0, x)=\widetilde{\psi}_{0} \quad \text { in } \quad(c, d) .
$$

Therefore, the above system is conservative $i . e$.

$\mathcal{E}(\widetilde{\varphi}, \widetilde{\psi}, \widetilde{\Phi}, \widetilde{\Psi}, \widetilde{\theta}, \widetilde{u}, \widetilde{v}, \widetilde{p}, \widetilde{q}, \widetilde{z}, \widetilde{w}, \widetilde{r}, \widetilde{y})(t)=\mathcal{E}(\widetilde{\varphi}, \widetilde{\psi}, \widetilde{\Phi}, \widetilde{\Psi}, \widetilde{\theta}, \widetilde{u}, \widetilde{v}, \widetilde{p}, \widetilde{q}, \widetilde{z}, \widetilde{w}, \widetilde{r}, \widetilde{y})(0), \quad \forall t>0$

Note that, in $\Theta$ the problem is purely thermoelastic. However, in $(0, L) \backslash \Theta$ the energy is conservative i.e.

$$
\mathcal{E}_{1}(t)=\mathcal{E}_{1}(0), \quad \forall t>0
$$

Therefore, the semigroup $T$ associated to (3.18), (3.19), and (3.20), defined on the Hilbert space

$$
\mathcal{H}_{0}:=\left(H_{0}^{1}(] c, d[)\right)^{2} \times(\{0\})^{3} \times\left(L^{2}(] c, d[)\right)^{2} \times(\{0\})^{2} \times\left(\left\{0_{\mathbb{C}}\right\}\right)^{4}
$$

by

$$
S_{0}\left(\widetilde{\varphi}_{0}, \widetilde{\psi}_{0}, 0,0,0, \widetilde{\varphi}_{1}, \widetilde{\psi}_{1}, 0,0,0,0,0,0\right):=\left(\widetilde{\varphi}, \widetilde{\psi}, 0,0,0, \widetilde{\varphi}_{t}, \widetilde{\psi}_{t}, 0,0,0,0,0,0\right)
$$

is a unitary semigroup. Hence, $r_{\mathrm{ess}}\left(S_{0}(t)\right)=1$. We attract the attention of the readers to that in this case, the problem is purely elastic in $(c, d)$.

Theorem 3.2. The semigroup $S$ with $S(t)=e^{\mathcal{A} t}$ is not exponentially stable.

Proof. Firstly to choose a Hilbert space $\mathcal{H}_{0}$, we proceed as Rivera and Racke in [18]. Let $\left(\varphi_{0}^{n}, \psi_{0}^{n}, 0,0,0, \varphi_{1}^{n}, \psi_{1}^{n}, 0,0\right)$ be a bounded sequence of initial data in

$$
\mathcal{H}_{0}:=\left(H_{0}^{1}(] c, d[)\right)^{2} \times(\{0\})^{3} \times\left(L^{2}(] c, d[)\right)^{2} \times(\{0\})^{2} .
$$

Let $\left(\varphi^{n}, \psi^{n}, \Phi^{n}, \Psi^{n}, \theta^{n}\right)$ be the corresponding solution to the transmission problem (1.1)-(1.2) with associated semigroup $S(t)=e^{\mathcal{A} t}$ and let $\left(\widetilde{\varphi}^{n}, \widetilde{\psi}^{n}\right)$ be the solution of the uncoupled system $(3.18)-(1.3)$ with $\left(\widetilde{\Phi}^{n}, \widetilde{\Psi}^{n}, \widetilde{\theta}^{n}\right):=(0,0,0)$ with associated semigroup $S_{0}(t)=e^{\widetilde{\mathcal{A}} t}$. Let

$$
\begin{aligned}
& \chi^{n}:=\varphi^{n}-\widetilde{\varphi}^{n}, \zeta^{n}:=\psi^{n}-\widetilde{\psi}^{n}, \eta^{n}:=\Phi^{n}-\widetilde{\Phi}^{n}=\Phi^{n}, \\
& \xi^{n}:=\Psi^{n}-\widetilde{\Psi}^{n}=\Psi^{n}, \sigma:=\theta^{n}-\widetilde{\theta}^{n}=\theta^{n} .
\end{aligned}
$$

Our claim is to show that there exists a subsequence of $\left(\chi^{n}, \zeta^{n}, \eta^{n}, \xi^{n}, \sigma^{n}\right)$ converges in $\mathcal{H}$. 
Therefore $\left(\chi^{n}, \zeta^{n}, \eta^{n}, \xi^{n}, \sigma^{n}\right)$ satisfy the following system

$$
\left\{\begin{array}{l}
\rho_{1} \chi_{t t}^{n}-k\left(\chi_{x}^{n}+\zeta^{n}\right)_{x}=0 \quad \text { in }(0, L) \backslash \Theta \times(0, \infty) \\
\rho_{2} \zeta_{t t}^{n}-b \zeta_{x x}^{n}+k\left(\chi_{x}^{n}+\zeta^{n}\right)=0 \quad \text { in }(0, L) \backslash \Theta \times(0, \infty) \\
\rho_{1} \eta_{t t}^{n}-k\left(\eta_{x}^{n}+\xi^{n}\right)_{x}=0 \quad \text { in } \Theta \times(0, \infty) \\
\rho_{2} \xi_{t t}^{n}-b \xi_{x x}^{n}+k\left(\eta_{x}^{n}+\xi^{n}\right)+\alpha \sigma_{x}^{n}=0 \quad \text { in } \Theta \times(0, \infty) \\
\sigma_{t}^{n}-\sigma_{x x}^{n}+\alpha \xi_{x t}^{n}=0 \quad \text { in } \Theta \times(0, \infty) \\
\left(\eta^{n}, \eta_{t}^{n}, \xi^{n}, \xi_{t}^{n}, \sigma^{n}\right)(., 0)=(0,0,0,0,0) \text { in } \Theta \\
\left(\chi^{n}, \chi_{t}^{n}, \zeta^{n}, \zeta_{t}^{n}\right)(., 0)=(0,0,0,0) \text { in }(0, L) \backslash \Theta
\end{array}\right.
$$

The corresponding boundary conditions satisfied

$$
\left\{\begin{array}{l}
\eta^{n}(t, x)=\xi^{n}(t, x)=\sigma^{n}(t, x)=0, x=0, L \\
\eta^{n}(t, x)=\Phi^{n}(t, x), \xi^{n}(t, x)=\Psi^{n}(t, x), \sigma^{n}(t, x)=\theta^{n}(t, x), x=c, d \\
\chi^{n}(t, x)=\varphi^{n}(t, x), \zeta^{n}(t, x)=\psi^{n}(t, x), \quad(x=c, d)
\end{array}\right.
$$

For the associated energy to (3.22)-(3.23)

$$
\left\{\begin{aligned}
\mathcal{E}_{n}(t)= & \frac{1}{2} \int_{c}^{d}\left(\rho_{1}\left|\chi_{t}^{n}\right|^{2}+\rho_{2}\left|\zeta_{t}^{n}\right|^{2}+b\left|\zeta_{x}^{n}\right|^{2}+k\left|\chi_{x}+\zeta\right|^{2}\right) d x \\
& +\frac{1}{2} \int_{\Theta}\left(\rho_{1}\left|\eta_{t}^{n}\right|^{2}+\rho_{2}\left|\xi_{t}^{n}\right|^{2}+b\left|\xi_{x}^{n}\right|^{2}+k\left|\eta_{x}^{n}+\xi^{n}\right|^{2}+\left|\sigma^{n}\right|^{2}\right) d x
\end{aligned}\right.
$$

by a straightforward calculation, we have the identity

$$
\left\{\begin{aligned}
\frac{d}{d t} \mathcal{E}_{n}(t)+ & \frac{1}{2} \int_{\Theta}\left|\sigma_{x}\right|^{2} d x=\left.k\left(\chi_{x}+\zeta\right) \chi_{t}\right|_{c} ^{d}-\left.b \zeta_{x} \zeta_{t}\right|_{c} ^{d}+\left.k\left(\eta_{x}+\xi\right) \eta_{t}\right|_{0} ^{c} \\
& +\left.k\left(\eta_{x}+\xi\right) \eta_{t}\right|_{d} ^{L}-\left.b \xi_{x} \xi_{t}\right|_{0} ^{c}-\left.b \xi_{x} \xi_{t}\right|_{d} ^{L}+\left.\alpha \xi_{t} \sigma\right|_{0} ^{c}+\left.\alpha \xi_{t} \sigma\right|_{d} ^{L} \\
& +\left.\sigma_{x} \sigma\right|_{0} ^{c}+\left.\sigma_{x} \sigma\right|_{d} ^{L}
\end{aligned}\right.
$$

Hence, using the conditions (3.23) in (3.25) we get

$$
\left\{\begin{array}{l}
\frac{d}{d t} \mathcal{E}_{n}(t)+\frac{1}{2} \int_{\Theta}\left|\sigma_{x}\right|^{2} d x=k\left(\varphi_{x}+\psi\right)(d) \varphi_{t}(d)-k\left(\varphi_{x}+\psi\right)(c) \varphi_{t}(c) \\
-\widetilde{\varphi}_{x}(d) \varphi_{t}(d)+\widetilde{\varphi}_{x}(c) \varphi_{t}(c)-b \psi_{x}(d) \psi_{t}(d)-b \widetilde{\psi}_{x}(d) \psi_{t}(d) \\
+b \psi_{x}(c) \psi_{t}(c)+b \widetilde{\psi}_{x}(c) \psi_{t}(c)+k\left(\Phi_{x}+\Psi\right)(c) \Phi_{t}(c)-k \widetilde{\Phi}_{x}(c) \Phi_{t}(c) \\
-k\left(\Phi_{x}+\Psi\right)(d) \Phi_{t}(d)+\widetilde{\Phi}_{x}(d) \Phi_{t}(d)-b \Psi_{x}(c) \Psi_{t}(c)+b \widetilde{\Psi}_{x}(c) \Psi_{t}(c) \\
+b \Psi_{x}(d) \Psi_{t}(d)-b \widetilde{\Psi}_{x}(d) \Psi_{t}(d)+\alpha \Psi_{t}(c) \theta(c)-\alpha \Psi_{t}(d) \theta(d) \\
+\widetilde{\theta}_{x}(c) \theta(c)+\widetilde{\theta}_{x}(d) \theta(d)
\end{array}\right.
$$


Thanks to the fact that $\left(\widetilde{\Phi}^{n}, \widetilde{\Psi}^{n}, \widetilde{\theta}^{n}\right):=(0,0,0),(3.26)$ implies

$$
\left\{\begin{array}{l}
\frac{d}{d t} \mathcal{E}_{n}(t)+\frac{1}{2} \int_{\Theta}\left|\sigma_{x}\right|^{2} d x=k\left(\varphi_{x}+\psi\right)(d) \varphi_{t}(d)-k\left(\varphi_{x}+\psi\right)(c) \varphi_{t}(c) \\
-\widetilde{\varphi}_{x}(d) \varphi_{t}(d)+\widetilde{\varphi}_{x}(c) \varphi_{t}(c)-b \psi_{x}(d) \psi_{t}(d)-b \widetilde{\psi}_{x}(d) \psi_{t}(d) \\
+b \psi_{x}(c) \psi_{t}(c)+b \widetilde{\psi}_{x}(c) \psi_{t}(c)+k\left(\Phi_{x}+\Psi\right)(c) \Phi_{t}(c) \\
-k\left(\Phi_{x}+\Psi\right)(d) \Phi_{t}(d)-b \Psi_{x}(c) \Psi_{t}(c) \\
+b \Psi_{x}(d) \Psi_{t}(d)+\alpha \Psi_{t}(c) \theta(c)-\alpha \Psi_{t}(d) \theta(d)
\end{array}\right.
$$

Therefore, integrating over $[0, T]$, we obtain (3.28)

$$
\left\{\begin{array}{l}
\mathcal{E}_{n}(t) \leq k \int_{0}^{T}\left(\varphi_{x}+\psi\right)(d) \varphi_{t}(d) d t-k \int_{0}^{T}\left(\varphi_{x}+\psi\right)(c) \varphi_{t}(c) d t \\
-\int_{0}^{T} \widetilde{\varphi}_{x}(d) \varphi_{t}(d) d t+\int_{0}^{T} \widetilde{\varphi}_{x}(c) \varphi_{t}(c) d t-b \int_{0}^{T} \psi_{x}(d) \psi_{t}(d) d t-b \int_{0}^{T} \widetilde{\psi}_{x}(d) \psi_{t}(d) d t \\
+b \int_{0}^{T} \psi_{x}(c) \psi_{t}(c)+b \int_{0}^{T} \widetilde{\psi}_{x}(c) \psi_{t}(c)+k \int_{0}^{T}\left(\Phi_{x}+\Psi\right)(c) \Phi_{t}(c) \\
-k \int_{0}^{T}\left(\Phi_{x}+\Psi\right)(d) \Phi_{t}(d) d t-b \int_{0}^{T} \Psi_{x}(c) \Psi_{t}(c) d t \\
+b \int_{0}^{T} \Psi_{x}(d) \Psi_{t}(d) d t+\alpha \int_{0}^{T} \Psi_{t}(c) \theta(c) d t-\alpha \int_{0}^{T} \Psi_{t}(d) \theta(d) d t .
\end{array}\right.
$$

Due to (1.3) the sequences $\varphi_{t}^{n}(c), \varphi_{t}^{n}(d), \psi_{t}^{n}(c)$, and $\psi_{t}^{n}(d)$ are bounded in $H^{1}(0, T)$. Therefore, the sequence $\left(\chi^{n}, \zeta^{n}, \eta^{n}, \xi^{n}, \sigma^{n}\right)$ has a convergent subsequence in $L^{2}(0, T)$. Hence, $S(t)-S_{0}(t)$ is compact.

\section{Polynomial Stabilisation}

Lemma 4.1. There is no eigenvalue on the imaginary axis i.e. we have

$$
i \mathbb{R} \subset \rho(\mathcal{A}),
$$

Proof. Suppose that there exists $\lambda \in \mathbb{R}^{*}$ such that eigenvalue and

$$
W=\left(\varphi, \psi, \Phi, \Psi, \theta, u, v, p, q, z_{1}, z_{2}, r_{1}, r_{2}\right)
$$

be the normalized eigenfunction, i.e.,

$$
\mathcal{A} W=i \lambda W .
$$


Therefore, we have

$$
\begin{gathered}
u(x)=i \lambda \varphi(x), \quad x \in(c, d), \\
v(x)=i \lambda \psi(x), \quad x \in(c, d), \\
p(x)=i \lambda \Phi(x), \quad x \in \Theta, \\
q(x)=i \lambda \Psi(x), \quad x \in \Theta, \\
\theta_{x x}-\alpha q_{x}=i \lambda \theta(x), \quad x \in \Theta
\end{gathered}
$$

and so we have

$$
\begin{gathered}
\lambda^{2} \varphi+\frac{k}{\rho_{1}}\left(\varphi_{x x}+\psi_{x}\right)=0, \quad x \in(c, d) \\
\lambda^{2} \psi+\frac{b}{\rho_{2}} \psi_{x x}-\frac{k}{\rho_{2}}\left(\varphi_{x}+\psi\right)=0, \quad x \in(c, d) \\
\lambda^{2} \Phi+\frac{k}{\rho_{1}}\left(\Phi_{x x}+\Psi_{x}\right)=0, \quad x \in \Theta \\
\lambda^{2} \Psi+\frac{b}{\rho_{2}} \Psi_{x x}-\frac{k}{\rho_{2}}\left(\Phi_{x}+\Psi\right)-\frac{\alpha}{\rho_{2}} \theta_{x}=0, \quad x \in \Theta \\
-M \lambda^{2} \Phi(c)=k\left(\Phi_{x}+\Psi\right)(c)-k\left(\varphi_{x}+\psi\right)(c), \\
-M \lambda^{2} \Psi(c)=b \Psi_{x}(c)-\alpha \theta(c)-b \psi_{x}(c), \\
N \lambda^{2} \Phi(d)=k\left(\Phi_{x}+\Psi\right)(d)-k\left(\varphi_{x}+\psi\right)(d), \\
N \lambda^{2} \Psi(d)=b \Psi_{x}(d)-\alpha \theta(d)-b \psi_{x}(d) .
\end{gathered}
$$

We have

$$
\operatorname{Re}\langle\mathcal{A} W, W\rangle_{\mathcal{H}}=\operatorname{Re}\left(i \lambda\|W\|_{\mathcal{H}}^{2}\right)=-\int_{\Theta}\left|\theta_{x}\right|^{2} d x=0 .
$$

So, $\theta_{x}=0$ on $\Theta$. In addition, by $(1.2)$ we have $\theta(0)=\theta(L)=0$. Hence, $\theta=0$ on $\Theta$. Next, thanks to $(4.26), q_{x}=0$ on $\Theta$. Thus, $q=0$ on $\Theta$ because $q(0)=q(L)=$ 0 . Therefore, (4.32) leads to $\Psi=0$ on $\Theta$. Then, by (4.11) and the conditions $\Phi(0)=\Phi(L)=0$ we get $\Phi=0$ on $\Theta$. Next, the transmission conditions, (4.12), $(4.13),(4.14)$, and $(4.15)$ give

$$
\varphi(x)=\varphi_{x}(x)=\psi(x)=\psi_{x}(x), \quad x=c, d .
$$

Now, denote by $Y=\left(\varphi, \varphi_{x}, \psi, \psi_{x}\right)$. then from equations $(4.33),(4.34)$, and the boundary conditions (4.17), we obtain the following system

$$
Y^{\prime}=M Y \quad \text { and } \quad Y(c)=Y(d)=0,
$$

where $Y$ is is a real square matrix of order three. Then the ordinary differential system (4.18) has the unique solution $Y=0$. Consequently, $W=0$ which contradicts that $W \neq 0$.

For the polynomial stabilisation, the following theorem introduced by B. Rao and Z.Liu [11] will be used.

Theorem 4.1. there exist two constants $C_{m}>0$ independent of $W^{0} \in D\left(\mathcal{A}^{m}\right)$ such that

$$
\left\|S(t) W^{0}\right\|_{\mathcal{H}} \leq C_{m}\left(\frac{\ln t}{t}\right)^{\frac{m}{48}}(\ln t)\left\|W^{0}\right\|_{D(\mathcal{A})}, \quad m \in \mathbb{N}^{*}
$$


Proof. To conclude the mentioned polynomial stability, we must prove the following two conditions:

$$
i \mathbb{R} \subset \rho(\mathcal{A}),
$$

and

$$
\lim _{\lambda \longrightarrow \infty} \frac{1}{\lambda^{48}}\left\|(i \lambda I-\mathcal{A})^{-1}\right\|<+\infty .
$$

We will establish these conditions by contradictions. The proof of the condition (4.20) is standard.

Now, suppose that the condition (4.21) is false. Then, there is a real sequence $\left(\lambda_{n}\right)$ and a sequence

$$
W^{n}=\left(\varphi^{n}, \psi^{n}, \Phi^{n}, \Psi^{n}, \theta^{n}, u^{n}, v^{n}, p^{n}, q^{n}, z_{1}^{n}, z_{2}^{n}, r_{1}^{n}, r_{2}^{n}\right) \in D(\mathcal{A})
$$

such that

$$
\begin{aligned}
& \left|\lambda_{n}\right| \longrightarrow+\infty, \\
& \left\|W^{n}\right\|_{\mathcal{H}}=1,
\end{aligned}
$$

and

$$
\lim _{n \longrightarrow \infty} \lambda_{n}^{48}\left\|\left(i \lambda_{n} I-\mathcal{A}\right) W^{n}\right\|_{\mathcal{H}}=0 .
$$

So

$$
\lambda_{n}^{48}\left[\left(i \lambda_{n} I-\mathcal{A}\right)\right] W^{n}=\left(f_{1}^{n}, f_{2}^{n}, \ldots, f_{9}^{n}, f_{10}, f_{11}, f_{12}, f_{13}\right) \longrightarrow 0 \quad \text { in } \quad \mathcal{H},
$$

Therefore, in $H^{1}(] c, d[)$ we have the following convergence

$$
\begin{aligned}
\lambda_{n}^{48}\left[i \lambda_{n} \varphi^{n}-u^{n}\right] & =f_{1}^{n} \longrightarrow 0, \\
\lambda_{n}^{48}\left[i \lambda_{n} \psi^{n}-v^{n}\right] & =f_{2}^{n} \longrightarrow 0,
\end{aligned}
$$

In $H^{1}(\Theta)$ we have the following convergence

$$
\begin{aligned}
& \lambda_{n}^{48}\left[i \lambda_{n} \Phi^{n}-p^{n}\right]=f_{3}^{n} \longrightarrow 0, \\
& \lambda_{n}^{48}\left[i \lambda_{n} \Psi^{n}-q^{n}\right]=f_{4}^{n} \longrightarrow 0,
\end{aligned}
$$

In $L^{2}(\Theta)$ we have the following convergence

$$
\lambda_{n}^{48}\left[i \lambda_{n} \theta^{n}-\theta_{x x}^{n}+\alpha q_{x}^{n}\right]=f_{5}^{n} \longrightarrow 0 .
$$

In $L^{2}(] c, d[)$ we have the following convergence

$$
\begin{gathered}
\lambda_{n}^{48}\left[i \lambda_{n} u^{n}-\frac{k}{\rho_{1}}\left(\varphi_{x x}^{n}+\psi_{x}^{n}\right)\right]=f_{6}^{n} \longrightarrow 0, \\
\lambda_{n}^{48}\left[i \lambda_{n} v^{n}-\frac{b}{\rho_{2}} \psi_{x x}^{n}+\frac{k}{\rho_{2}}\left(\varphi_{x}^{n}+\psi^{n}\right)\right]=f_{7}^{n} \longrightarrow 0,
\end{gathered}
$$

In $L^{2}(\Theta)$ we have the following convergence

$$
\begin{gathered}
\lambda_{n}^{48}\left[i \lambda_{n} p^{n}-\frac{k}{\rho_{1}}\left(\Phi_{x x}^{n}+\Psi_{x}^{n}\right)\right]=f_{8}^{n} \longrightarrow 0, \\
\lambda_{n}^{48}\left[i \lambda_{n} q^{n}-\frac{b}{\rho_{2}} \Psi_{x x}^{n}+\frac{k}{\rho_{2}}\left(\Phi_{x}^{n}+\Psi^{n}\right)+\frac{\alpha}{\rho_{2}} \theta_{x}^{n}\right]=f_{9}^{n} \longrightarrow 0,
\end{gathered}
$$


Also, In $\mathbb{C}$ we have the following convergence

$$
\begin{gathered}
\lambda_{n}^{48}\left[i \lambda_{n} z_{1}^{n}+\frac{1}{M}\left(k\left(\Phi_{x}^{n}+\Psi^{n}\right)(c)-k\left(\varphi_{x}^{n}+\psi^{n}\right)(c)\right)\right]=f_{10}^{n} \longrightarrow 0, \\
\lambda_{n}^{48}\left[i \lambda_{n} z_{2}^{n}+\frac{1}{M}\left(b \Psi_{x}^{n}(c)-\alpha \theta^{n}(c)-b \psi_{x}^{n}(c)\right)\right]=f_{11}^{n} \longrightarrow 0, \\
\lambda_{n}^{48}\left[i \lambda_{n} r_{1}^{n}+\frac{1}{N}\left(-k\left(\Phi_{x}^{n}+\Psi^{n}\right)(d)+k\left(\varphi_{x}^{n}+\psi^{n}\right)(d)\right)\right]=f_{12}^{n} \longrightarrow 0,
\end{gathered}
$$

and

$$
\lambda_{n}^{48}\left[i \lambda_{n} r_{2}^{n}+\frac{1}{N}\left(-b \Psi_{x}^{n}(d)+\alpha \theta^{n}(d)-b \psi_{x}^{n}(d)\right)\right]=f_{13}^{n} \longrightarrow 0,
$$

Note that

$$
\begin{aligned}
\left\|W^{n}\right\|_{\mathcal{H}}^{2}= & \int_{c}^{d}\left[\rho_{1}\left|u^{n}\right|^{2}+\rho_{2}\left|v^{n}\right|^{2}+b\left|\psi_{x}^{n}\right|^{2}+k\left|\varphi_{x}^{n}+\psi\right|^{2}\right] d x \\
& +\int_{\Theta}\left[\rho_{1}\left|p^{n}\right|^{2}+\rho_{2}\left|q^{n}\right|^{2}+b\left|\Psi_{x}^{n}\right|^{2}+k\left|\Phi_{x}^{n}+\Psi^{n}\right|^{2}+\left|\theta^{n}\right|^{2}\right] d x \\
& +\left|z_{1}^{n}\right|^{2}+\left|z_{2}^{n}\right|^{2}+\left|r_{1}^{n}\right|^{2}+\left|r_{2}^{n}\right|^{2} .
\end{aligned}
$$

The main goal is to prove that $\left\|W^{n}\right\|_{\mathcal{H}}^{2} \longrightarrow 0$ to get a contradiction with (4.23). On the other side, we have The proof will contains several steps.

Step 1. Thanks to (4.25), we obtain

$$
\operatorname{Re}\left\langle\left(\lambda_{n}^{48}\left(i \lambda_{n}-\mathcal{A}\right)\right) W^{n}, W^{n}\right\rangle_{\mathcal{H}}=\int_{\Theta}\left|\lambda_{n}^{24} \theta_{x}^{n}\right|^{2} d x \longrightarrow 0
$$

Hence

$$
\lambda_{n}^{24} \theta_{x}^{n} \longrightarrow 0 \quad \text { in } \quad L^{2}(\Theta) .
$$

Thanks to the Poincaré inequality, we have also

$$
\lambda_{n}^{24} \theta^{n} \longrightarrow 0 \quad \text { in } \quad L^{2}(\Theta) .
$$

Step 2. In this step, to achieve the proof, we shall establish that

$$
\begin{array}{rlll}
u^{n}, v^{n} & \longrightarrow 0 & \text { in } & L^{2}(c, d), \\
\psi_{x}^{n} & \longrightarrow 0 & \text { in } & L^{2}(c, d), \\
\varphi_{x}^{n}+\psi^{n} & \longrightarrow 0 & \text { in } & L^{2}(c, d),
\end{array}
$$

Firstly, dividing (4.26), (4.27), (4.28), and (4.29) by $\lambda_{n}^{49}$ and using the fact that $u^{n}$, $v^{n}, z^{n}, p^{n}, q^{n}$, and $r^{n}$ are bounded from (4.23) lead to

$$
\begin{array}{lll}
\varphi^{n}, \psi^{n} \longrightarrow 0 \quad \text { in } & L^{2}(c, d) . \\
\Phi^{n}, \Psi^{n} \longrightarrow 0 & \text { in } & L^{2}(\Theta) .
\end{array}
$$

Next, eliminating $u^{n}$ in (4.31) by (4.26) then taking the inner product of resulting equation with $\frac{\rho_{1}}{\lambda_{n}^{48}}(c-x) \varphi_{x}^{n}$ in $L^{2}(c, d)$ and integrating by parts lead to

$$
\left\{\begin{array}{l}
-\frac{\rho_{1}}{2} \int_{c}^{d}\left|\lambda_{n} \varphi^{n}\right|^{2}+\rho_{1} \frac{c-d}{2}\left|\lambda_{n} \varphi^{n}(d)\right|^{2}-\frac{k}{2} \int_{c}^{d}\left|\varphi_{x}^{n}\right|^{2}+\frac{k}{2}\left|\varphi_{x}^{n}(d)\right|^{2} \\
-k \int_{c}^{d}(c-x) \psi_{x}^{n} \varphi_{x}^{n} \longrightarrow 0 .
\end{array}\right.
$$


The sequence $\left\|\varphi_{x}^{n}\right\|_{L^{2}(c, d)}$ is bouded. Indeed,

$$
\left\|\varphi_{x}^{n}\right\|_{L^{2}(c, d)} \leq\left\|\varphi_{x}^{n}+\psi^{n}\right\|_{L^{2}(c, d)}+\left\|\psi^{n}\right\|_{L^{2}(c, d)},
$$

$\left\|\varphi_{x}^{n}+\psi^{n}\right\|_{L^{2}(c, d)}$ is bounded from (4.23) and $\left\|\psi^{n}\right\|_{L^{2}(c, d)}$ is bounded from (4.45). Therefore, the last term in (4.47) converges to zero thanks to (4.45). Thus, (4.47) gives

$$
-\frac{\rho_{1}}{2} \int_{c}^{d}\left|\lambda_{n} \varphi^{n}\right|^{2}+\rho_{1} \frac{c-d}{2}\left|\lambda_{n} \varphi^{n}(d)\right|^{2}-\frac{k}{2} \int_{c}^{d}\left|\varphi_{x}^{n}\right|^{2}+\frac{k}{2}\left|\varphi_{x}^{n}(d)\right|^{2} \longrightarrow 0 .
$$

Similarly, eliminating $v^{n}$ in (4.32) by (4.27), then taking the inner product of resulting equations with $\frac{\rho_{2}}{\lambda_{n}^{48}}(c-x) \psi_{x}^{n}$ in $L^{2}(c, d)$ and integrating by parts lead to

$$
\left\{\begin{array}{l}
-\frac{\rho_{2}}{2} \int_{\mathcal{G}}^{d}\left|\lambda_{n} \psi^{n}\right|^{2}+\rho_{2} \frac{c-d}{2}\left|\lambda_{n} \psi^{n}(d)\right|^{2}-\frac{b}{2} \int_{c}^{d}\left|\psi_{x}^{n}\right|^{2}+\frac{b}{2}\left|\psi_{x}^{n}(d)\right|^{2} \\
+k \int_{c}^{d}(c-x) \psi_{x}^{n} \varphi_{x}^{n}+k \int_{c}^{d}(c-x) \psi_{x}^{n} \varphi^{n} \longrightarrow 0
\end{array}\right.
$$

The last term in (4.49) converges to zero due to (4.45) and the fact that $\left\|\psi_{x}^{n}\right\|_{L^{2}(c, d)}$ is bounded by (4.23). Hence (4.49) leads to

$$
-\frac{\rho_{2}}{2} \int_{c}^{d}\left|\lambda_{n} \psi^{n}\right|^{2}+\rho_{2} \frac{c-d}{2}\left|\lambda_{n} \psi^{n}(d)\right|^{2}-\frac{b}{2} \int_{c}^{d}\left|\psi_{x}^{n}\right|^{2}+\frac{b}{2}\left|\psi_{x}^{n}(d)\right|^{2} \longrightarrow 0 .
$$

Adding (4.48) and (4.50) leads to

$$
\left\{\begin{array}{l}
-\frac{\rho_{1}}{2} \int_{c}^{d}\left|\lambda_{n} \varphi^{n}\right|^{2}-\frac{\rho_{2}}{2} \int_{c}^{d}\left|\lambda_{n} \psi^{n}\right|^{2}-\frac{k}{2} \int_{c}^{d}\left|\varphi_{x}^{n}\right|^{2}-\frac{b}{2} \int_{c}^{d}\left|\psi_{x}^{n}\right|^{2} \\
+\rho_{1} \frac{c-d}{2}\left|\lambda_{n} \varphi^{n}(d)\right|^{2}+\rho_{2} \frac{c-d}{2}\left|\lambda_{n} \psi^{n}(d)\right|^{2}+\frac{k}{2}\left|\varphi_{x}^{n}(d)\right|^{2} \\
\quad+\frac{b}{2}\left|\psi_{x}^{n}(d)\right|^{2} \longrightarrow 0
\end{array}\right.
$$

In the following steps and calculation, we shall denote by $\|\cdot\|_{d}:=\|\cdot\|_{L^{2}(d, L)}$.

Step 3. Our target in this step is to prove that all the terms defined at $x=d$ in (4.51) tend to zero. Only, the transmission conditions will give back information from the thermoelastic part of the beam to the elastic part. Let us start with the term $\lambda_{n} \psi^{n}(d)$. So by the Gagliardo-Nirenberg inequality, we have

$$
\left|\lambda_{n} \psi^{n}(d)\right|^{2}=\left|\lambda_{n} \Psi^{n}(d)\right|^{2} \lesssim\left\|\Psi^{n}\right\|_{d}\left\|\lambda_{n} \Psi_{x}^{n}\right\|_{d} .
$$

Eliminating $q_{n}$ in (4.30) by (4.29) then taking the inner product of resulting equation with $\frac{1}{\lambda_{n}^{37}} \Psi_{x}^{n}$ in $L^{2}(d, L)$ and integrating by parts lead to

$$
i \int_{d}^{L} \lambda_{n}^{12} \theta^{n} \Psi_{x}^{n}+\int_{d}^{L} \lambda_{n}^{11} \theta_{x}^{n} \Psi_{x x}^{n}-\left[\lambda_{n}^{11} \theta_{x}^{n} \Psi_{x}^{n}\right]_{d}^{L}+i \alpha \int_{d}^{L}\left|\lambda_{n}^{6} \Psi_{x}^{n}\right|^{2} \longrightarrow 0 .
$$

The first term tends to zero since $\lambda_{n}^{12} \theta^{n} \longrightarrow 0$ by (4.40) and $\Psi_{x}^{n}$ is bounded in $L^{2}(d, L)$ due to (4.23). The second term can be written as

$$
\int_{d}^{L} \lambda_{n}^{12} \theta_{x}^{n} \frac{\Psi_{x x}^{n}}{\lambda_{n}} .
$$


So this term tends to zero since $\frac{\Psi_{x x}^{n}}{\lambda_{n}}$ is bounded from the dividing of (4.34) by $\lambda_{n}^{49}$ and $\lambda_{n}^{12} \theta_{x}^{n}$ tends to zero in $L^{2}(d, L)$ by (4.40). About the boundary term, using the transmission conditions on $\theta_{x}^{n}$ gives

$$
\left[\lambda_{n}^{11} \theta_{x}^{n} \Psi_{x}^{n}\right]_{d}^{L}=-\lambda_{n}^{11} \theta_{x}^{n}(L) \Psi_{x}^{n}(L) .
$$

By the Gagliardo-Nirenberg inequality we have

$$
\left|\lambda_{n}^{11} \theta_{x}^{n}(L) \Psi_{x}^{n}(L)\right| \lesssim\left\|\lambda_{n}^{24} \theta_{x}^{n}\right\|_{d}^{\frac{1}{2}}\left\|\frac{\theta_{x x}^{n}}{\lambda_{n}}\right\|_{d}^{\frac{1}{2}}\left\|\Psi_{x}^{n}\right\|_{d}^{\frac{1}{2}}\left\|\frac{\Psi_{x x}^{n}}{\lambda_{n}}\right\|_{d}^{\frac{1}{2}} .
$$

Now, dividing (4.29) by $\lambda_{n}^{9}$ implies that $\frac{q_{x}^{n}}{\lambda_{n}}$ is bounded in $L^{2}(d, L)$. Then, dividing (4.30) by $\lambda_{n}^{9}$ yields to $\frac{\theta_{x x}^{n}}{\lambda_{n}}$ is bounded in $L^{2}(d, L)$. Consequently, the first three terms in (4.53) tend to zero and so

$$
\left\|\lambda_{n}^{6} \Psi_{x}^{n}\right\|_{d} \longrightarrow 0
$$

Therefore,

$$
\left\|\lambda_{n} \Psi_{x}^{n}\right\|_{d} \longrightarrow 0 .
$$

On the other hand, $\Psi^{n}$ is bounded in $L^{2}(d, L)$ from the Poincaré inequality and since $\Psi_{x}^{n}$ is bounded in $L^{2}(d, L)$ from (4.23). Thus, using (4.55) in (4.52) leads to

$$
\lambda_{n} \psi^{n}(d) \longrightarrow 0 .
$$

Now, let us prove that

$$
\left\|\lambda_{n}\left(\Phi_{x}+\Psi^{n}\right)\right\|_{d} \longrightarrow 0 .
$$

For this goal, eliminating $q_{n}$ in (4.34) by (4.29) then taking the inner product of resulting equation with $\frac{1}{\lambda_{n}^{46}}\left(\Phi_{x}+\Psi^{n}\right)$ in $L^{2}(d, L)$ and integrating by parts lead to

$$
\left\{\begin{array}{l}
-\int_{d}^{L} \lambda_{n}^{4} \Psi^{n}\left(\Phi_{x}+\Psi^{n}\right)+\frac{b}{\rho_{2}} \int_{d}^{L} \lambda_{n}^{2} \Psi_{x}^{n}\left(\Phi_{x}+\Psi^{n}\right)_{x} \\
-\frac{b}{\rho_{2}}\left[\lambda_{n}^{2} \Psi_{x}^{n}\left(\Phi_{x}+\Psi^{n}\right)\right]_{d}^{L}+\frac{k}{\rho_{2}} \int_{d}^{L}\left|\lambda_{n}\left(\Phi_{x}^{n}+\Psi^{n}\right)\right|^{2} \\
+\frac{\alpha}{\rho_{2}} \int_{d}^{L} \lambda_{n}^{2} \theta_{x}^{n}\left(\Phi_{x}+\Psi^{n}\right) \longrightarrow 0 .
\end{array}\right.
$$

By the poincaré inequality we have

$$
\left\|\lambda_{n}^{4} \Psi^{n}\right\|_{d} \leq\left\|\lambda_{n}^{4} \Psi_{x}^{n}\right\|_{d}
$$

Hence, thanks to (4.54)

$$
\lambda_{n}^{4} \Psi^{n} \longrightarrow 0 \quad \text { in } \quad L^{2}(d, L) .
$$

So (4.59) and the boundedness of $\Phi_{x}+\Psi^{n}$ in $L^{2}(d, L)$ due to (4.23) give

$$
\int_{d}^{L} \lambda_{n}^{4} \Psi^{n}\left(\Phi_{x}+\Psi^{n}\right) \longrightarrow 0
$$

Next, we have

$$
\int_{d}^{L} \lambda_{n}^{2} \Psi_{x}^{n}\left(\Phi_{x}+\Psi^{n}\right)_{x}=\int_{d}^{L} \lambda_{n}^{3} \Psi_{x}^{n} \frac{\left(\Phi_{x}+\Psi^{n}\right)_{x}}{\lambda_{n}} \longrightarrow 0
$$


since $\lambda_{n}^{3} \Psi_{x}^{n} \longrightarrow 0$ in $L^{2}(d, L)$ thanks to $(4.54)$ and $\frac{\left(\Phi_{x}+\Psi^{n}\right)_{x}}{\lambda_{n}}$ is bounded in $L^{2}(d, L)$ by dividing (4.33) by $\frac{1}{\lambda_{n}^{49}}$. The last term in (4.58)

$$
\int_{d}^{L} \lambda_{n}^{2} \theta_{2 x}^{n}\left(\Phi_{x}+\Psi^{n}\right) \longrightarrow 0
$$

Thanks to (4.40) and the fact that $\Phi_{x}+\Psi^{n}$ is bounded due to (4.23). Now, about the boundary terms in (4.58), by the Gagliardo-Nirenberg inequality we have for $x=d, L$

$$
\left\{\begin{array}{l}
\left|\lambda_{n}^{2} \Psi_{x}^{n}(x)\left(\Phi_{x}+\Psi^{n}\right)(x)\right| \lesssim \\
\left\|\lambda_{n}^{6} \Psi_{x}^{n}\right\|_{d}^{\frac{1}{2}}\left\|\frac{\Psi_{x x}^{n}}{\lambda_{n}}\right\|_{d}^{\frac{1}{2}}\left\|\Phi_{x}+\Psi^{n}\right\|_{d}^{\frac{1}{2}}\left\|\frac{\left(\Phi_{x}+\Psi^{n}\right)_{x}}{\lambda_{n}}\right\|_{d}^{\frac{1}{2}}
\end{array}\right.
$$

Thus, (4.54) and the boundedness of $\frac{\Psi_{x x}^{n}}{\lambda_{n}}, \Phi_{x}+\Psi^{n}$, and $\frac{\left(\Phi_{x}+\Psi^{n}\right)_{x}}{\lambda_{n}}$ yield

$$
\left[\lambda_{n}^{2} \Psi_{x}^{n}\left(\Phi_{x}+\Psi^{n}\right)\right]_{d}^{L} \longrightarrow 0 .
$$

Consequently, (4.60), (4.61), (4.62), and (4.64) imply (4.57). Next, by the transmission condition and the Gagliardo-Nirenberg inequality, we have

$$
\left|\lambda_{n} \varphi^{n}(d)\right|=\left|\lambda_{n} \Phi^{n}(d)\right| \lesssim\left\|\lambda_{n} \Phi_{x}^{n}\right\|_{d}^{\frac{1}{2}}\left\|\lambda_{n} \Phi^{n}\right\|_{d}^{\frac{1}{2}} .
$$

Next, the Poincaré inequality leads to

$$
\left|\lambda_{n} \varphi^{n}(d)\right| \lesssim\left\|\lambda_{n} \Phi_{x}^{n}\right\|_{d}
$$

Therefore, the inequality

$$
\left\|\Phi_{x}\right\|_{d} \leq C\left(\left\|\Phi_{x}+\Psi^{n}\right\|_{d}+\left\|\Psi_{x}\right\|_{d}\right)
$$

leads to

$$
\left\|\lambda_{n} \Phi_{x}^{n}\right\|_{d} \longrightarrow 0
$$

and so (4.65) gives

$$
\left|\lambda_{n} \varphi^{n}(d)\right| \longrightarrow 0 .
$$

Step 4. In this step, the claim is the proving that the last two terms in (4.51) tend to zero. Firstly, by the transmission conditions (1.2) and the Gagliardo-Nirenberg inequality we have

$$
\left|\varphi^{n}(d)\right|=\left|\Phi^{n}(d)\right| \lesssim\left\|\Phi^{n}\right\|_{d}^{\frac{1}{2}}\left\|\Phi_{x}^{n}\right\|_{d}^{\frac{1}{2}}
$$

Thus, (4.67) gives

$$
\left|\varphi^{n}(d)\right| \longrightarrow 0 .
$$

Repeating the same process yields also

$$
\left|\psi^{n}(d)\right| \longrightarrow 0 .
$$

Dividing (4.26) and (4.27) by $\lambda^{48}$, the sequence $\lambda^{n}\left(\varphi_{x}^{n}+\psi^{n}\right)$ is bounded in $L^{2}(] c, d[)$. On the other side, dividing (4.31) by $\lambda_{n}^{49}$, the sequence $\frac{\left(\varphi_{x}^{n}+\psi^{n}\right)_{x}}{\lambda^{n}}$ is bounded in 
$L^{2}(] c, d[)$. By the Gagliardo-Nirenberg inequality we have

$$
\left|\left(\varphi_{x}^{n}+\psi^{n}\right)(d)\right| \lesssim\left\|\lambda^{n}\left(\varphi_{x}^{n}+\psi^{n}\right)\right\|_{d}^{\frac{1}{2}}\left\|\frac{\left(\varphi_{x}^{n}+\psi^{n}\right)_{x}}{\lambda^{n}}\right\|_{d}^{\frac{1}{2}} .
$$

Hence, the term $\left(\varphi_{x}^{n}+\psi^{n}\right)(d)$ is bounded in $\mathbb{C}$.

Repeating the same argument, we have $\left(\Phi_{x}^{n}+\Psi^{n}\right)(d)$ is bounded in $\mathbb{C}$. Therefore, dividing (4.37) by $\lambda_{n}^{49}$, we have

$$
r_{1}^{n} \longrightarrow 0 \text { in } \mathbb{C} .
$$

Moreover, thanks to (4.37) we have

$$
-\left(\Phi_{x}^{n}+\Psi^{n}\right)(d)+\left(\varphi_{x}^{n}+\psi^{n}\right)(d)=o(1) .
$$

Similarly, we prove that

$$
z_{1}^{n} \longrightarrow 0 \quad \text { in } \quad \mathbb{C}
$$

and

$$
\left(\Phi_{x}^{n}+\Psi^{n}\right)(c)-\left(\varphi_{x}^{n}+\psi^{n}\right)(c)=o(1) .
$$

Now, by the Gagliardo-Nirenberg inequality we have

$$
\left|\left(\Phi_{x}^{n}+\Psi^{n}\right)(d)\right| \lesssim\left\|\lambda^{n}\left(\Phi_{x}^{n}+\Psi^{n}\right)\right\|_{d}^{\frac{1}{2}}\left\|\frac{\left(\Phi_{x}^{n}+\Psi^{n}\right)_{x}}{\lambda^{n}}\right\|_{d}^{\frac{1}{2}}
$$

Since $\frac{\left(\Phi_{x}^{n}+\Psi^{n}\right)_{x}}{\lambda^{n}}$ is bounded in $L^{2}(d, L)$, using (4.57) in (4.75) gives

$$
\left|\left(\Phi_{x}^{n}+\Psi^{n}\right)(d)\right| \longrightarrow 0 .
$$

Therefore (4.72) gives

$$
\left(\varphi_{x}^{n}+\psi^{n}\right)(d) \longrightarrow 0 \quad \text { in } \quad \mathbb{C} .
$$

In addition,

$$
\left|\varphi_{x}^{n}(d)\right| \leq\left|\left(\varphi_{x}^{n}+\psi^{n}\right)(d)\right|+\left|\psi^{n}(d)\right| .
$$

Therefore,

$$
\left|\varphi_{x}^{n}(d)\right| \longrightarrow 0
$$

Similarly, we can prove that

$$
\left|\varphi_{x}^{n}(c)\right| \longrightarrow 0
$$

It remains to show that

$$
\left|\psi_{x}^{n}(d)\right|,\left|\psi_{x}^{n}(c)\right| \longrightarrow 0
$$

Dividing (4.27) by $\lambda^{48}$, the sequence $\lambda^{n} \psi_{x}^{n}$ ) is bounded in $L^{2}(] c, d[)$. On the other hand, dividing (4.32) by $\lambda_{n}^{49}$, the sequence $\frac{\psi_{x x}^{n}}{\lambda^{n}}$ is bounded in $L^{2}(] c, d[)$. Applying the Gagliardo-Nirenberg inequality we get

$$
\left|\psi_{x}^{n}(d)\right| \lesssim\left\|\lambda^{n} \psi_{x}^{n}\right\|_{d}^{\frac{1}{2}}\left\|\frac{\psi_{x x}^{n}}{\lambda^{n}}\right\|_{d}^{\frac{1}{2}} .
$$

Hence, the term $\psi_{x}^{n}(d)$ is bounded in $\mathbb{C}$.

Repeating the same argument, we have $\Psi_{x}^{n}(d)$ is bounded in $\mathbb{C}$. Therefore, dividing (4.38) by $\lambda_{n}^{49}$, we have

$$
r_{2}^{n} \longrightarrow 0 \quad \text { in } \quad \mathbb{C} \text {. }
$$


Moreover, thanks to (4.38) we have

$$
-b \Psi_{x}^{n}(d)+\alpha \theta^{n}(d)-b \psi_{x}^{n}(d)=o(1) .
$$

Same procedures lead to:

$$
z_{2}^{n} \longrightarrow 0 \text { in } \mathbb{C}
$$

and

$$
b \Psi_{x}^{n}(c)-\alpha \theta^{n}(c)+b \psi_{x}^{n}(c)=o(1) .
$$

Now, by the Gagliardo-Nirenberg inequality we have

$$
\left|\Psi_{x}^{n}(d)-\frac{\alpha}{b} \theta^{n}(d)\right| \lesssim\left\|\lambda^{n} \Psi_{x}^{n}\right\|_{d}^{\frac{1}{2}}\left\|\frac{\Psi_{x x}^{n}}{\lambda^{n}}\right\|_{d}^{\frac{1}{2}}+\left\|\theta^{n}\right\|_{d}^{\frac{1}{2}}\left\|\theta_{x}^{n}\right\|_{d}^{\frac{1}{2}} .
$$

Since $\frac{\Psi_{x x}^{n}}{\lambda^{n}}$ and $\theta_{x}^{n}$ are bounded in $L^{2}(d, L)$, using (4.41) and (4.55) in (4.85) gives

$$
\Psi_{x}^{n}(d)-\frac{\alpha}{b} \theta^{n}(d) \longrightarrow 0 \quad \text { in } \quad \mathbb{C} .
$$

So, due to (4.82), we obtain

$$
\psi_{x}^{n}(d) \longrightarrow 0 \quad \text { in } \quad \mathbb{C}
$$

Similarly, we prove that

$$
\psi_{x}^{n}(c) \longrightarrow 0 \quad \text { in } \quad \mathbb{C} .
$$

Hence, thanks to $(4.78),(4.79),(4.87)$, and (4.88), we get from (4.51) that

$$
-\frac{\rho_{1}}{2} \int_{c}^{d}\left|\lambda_{n} \varphi^{n}\right|^{2}-\frac{\rho_{2}}{2} \int_{c}^{d}\left|\lambda_{n} \psi^{n}\right|^{2}-\frac{k}{2} \int_{c}^{d}\left|\varphi_{x}^{n}\right|^{2}-\frac{b}{2} \int_{c}^{d}\left|\psi_{x}^{n}\right|^{2} \longrightarrow 0 .
$$

Consequently,

$$
\lambda_{n} \varphi^{n}, \lambda_{n} \psi^{n} \longrightarrow 0 \quad \text { in } \quad L^{2}(c, d)
$$

and

$$
\varphi_{x}^{n}, \psi_{x}^{n} \longrightarrow 0 \quad \text { in } \quad L^{2}(c, d) .
$$

Thus, (4.90), (4.26), and (4.27) imply that

$$
u^{n}, v^{n} \longrightarrow 0 \quad \text { in } \quad L^{2}(c, d) \text {. }
$$

Also, (4.91) with (4.45) leads to

$$
\varphi_{x}^{n}+\psi^{n}, \psi_{x}^{n} \longrightarrow 0 \text { in } L^{2}(c, d) .
$$

Moreover, since all results obtained on $L^{2}(0, c)$ are also obtained by the same way on $L^{2}(d, L)$, thanks to $(4.55)$ and $(4.57)$ we have

$$
\Phi_{x}^{n}+\Psi^{n}, \Psi_{x}^{n} \longrightarrow 0 \text { in } L^{2}(\Theta) .
$$

Also, the poincaré inequality, (4.55), and (4.57) give

$$
\lambda_{n} \Phi^{n}, \lambda_{n} \Psi^{n} \longrightarrow 0 \text { in } L^{2}(\Theta) .
$$

Therefore, by dividing (4.28) and (4.29) by $\lambda_{n}^{48}$ we get

$$
p^{n}, q^{n} \longrightarrow 0 \text { in } L^{2}(\Theta) .
$$

In addition, dividing (4.41) by $\lambda_{n}^{24}$ leads to

$$
\theta^{n} \longrightarrow 0 \text { in } L^{2}(\Theta) \text {. }
$$


Finally, (4.92), (4.93), (4.94), (4.96), and (4.97) imply

$$
\left\|W^{n}\right\|_{\mathcal{H}} \longrightarrow 0 \text {. }
$$

This a contradiction with (4.23) and the proof is achieved.

\section{REFERENCES}

[1] M.S. Alves, C. A. Raposo, J. E. Muñoz Rivera, M. Sepúlveda, O. V. Villagrán, Uniform stabilization for the transmission problem of the Timoshenko system with memory, J. Math. Anal. Appl. 369 (2010) 323-345.

[2] M. Aouadi, A. Soufyane, Decay of the timoshenko beam with thermal effect and memory boundary conditions, Journal of Dynamical and Control Systems (2013), Volume 19, pp 33-46.

[3] A. Borichev and Y. Tomilov, Optimal polynomial decay of functions and operator semigroups, Math. Ann., 347 (2009), pp. 455478.

[4] K. Engel, R. Nagel, One-Parameter Semigroups for Linear Evolution Equations. Graduate Texts in Mathematics. New York: Springer Verlag (2000).

[5] A. Haraux, Semi-groupes lineéaires et équations d'évolution linéaires peériodiques. Publication du Laboratoire d'Analyse Numeérique No. 78011, Universiteé Pierre et Marie Curie, Paris, 1978 .

[6] F. L. Huang, Strong asymptotic satbility of linear dynamical systems in Banach spaces, J. Diff. Equations 104 (1985), 307-324.

[7] Z-J. Han; G-Q Xu, Spectrum and stability analysis for a transmission problem in thermoelasticity with a concentrated mass, Z. Angew. Math. Phys. (2015).

[8] T. Kato, Pertubation Theory of Linear Operators. New York: Springer-Verlag (1980). .

[9] V. KOMORNIK, Exact controllability and stabilization. The Multiplier Method, RAM Res. Appl.Math., Masson, Paris, John Wiley, Chicester, UK, 1994.

[10] M. Kafini. General energy decay in a Timoshenko-type system of thermoelasticity of type III with a viscoelastic damping. J. Math. Anal. Appl. 375 (2011), 523537.

[11] Z. LIU; B. RAO, Characterization of polynomial decay rate for the solution of linear evolution equation, Z. Angew. Math. Phys. 56 (2005), 630-644.

[12] Z. Liu and S. Zheng, Semigroups Associated with Dissipative Systems, 398 Research Notes in Mathematics, Chapman \& Hall/CRC, 1999.

[13] T. K. Maryati; J. E. Muñoz Rivera; A. Rambaud; O. Vera, Stability of an $N$-component Timoshenko beam with localized Kelvin-Voigt and frictional dissipation, Electronic Journal of Differential Equations, Vol. (2018), pp. 1-18.

[14] S. A. Messaoudi; B. Said-Houari, energy decay in a transmission problem in thermoelasticity of type III, IMA Journal of Applied Mathematics (2009) 74, 344-360.

[15] S. A. Messaoudi and B. Said-Houari. Energy decay in a Timoshenko type system of thermoelasticity of type III. J. Math. Anal. Appl. 348 (2008), 298307.

[16] S. A. Messaoudi and B. Said-Houari. Energy decay in a Timoshenko type system with history in thermoelasticity of type III. Adv. Difference Equ. 4 (2009), 375400.

[17] J. Prüss, On the spectrum of $C_{0}$-semigroups, Trans. Amer. Math. Soc. 284 (1984), 847-857.

[18] J. E. Muñoz Rivera and R. Racke, Transmission Problems in (Thermo)Viscoelasticity with Kelvin-Voigt Damping: Nonexponential, Strong, and Polynomial Stability. SIAM J. Math. Analysis 49(5): 3741-3765 (2017).

[19] J. E. Muñoz Rivera; O. V. Villagran; M. Sepulveda, Stability to localized viscoelastic transmission problem, Communication in Part. Diff. Eq. (2018).

[20] C.A. Raposo; W.D. Bastos; M.L. Santosa, A transmission problem for the Timoshenko system, Computitional \& Applied Mathematics, Volume 26, pp. 215-234, (2007)

[21] A. Soufyane, M. Afilal, T. Aouam and M. Chacha. General decay of solutions of a linear one-dimesional porous-thermoelasaticity system with a boundary control of memory type. Nonlin. Anal. 72 (2010), 39033910.

[22] J. Wang; Z-J. Han; G-Q. Xu, Energy decay rate of transmission problem between thermoelasticity of type I and type II, Z. Angew. Math. Phys. (2017) 68-65.

[23] A. Wehbe and W. Youssef, Stabilization of the uniform Timoshenko beam by one locally distributed feedback, Applicable Analysis, 88 (2009), 1067-1078. 
[24] A. Wehbe and W. Youssef, Exponential and Polynomial Stability of an Elastic Bresse System with two Locally Distributed Feedbacks, Jornal of Math. Phy. JMP 51, 103523 (2010).

Wael Youssef, Department of mathematics, Faculty of Sciences 1, Lebanese UniVersity, Hadath, Beyrouth, Lebanon

E-mail address: Youssef.Wael@ul.edu.lb 